\title{
Spontaneous Retrobulbar Hemorrhage
}

David Clark Brewer, MD; Michael Mancera, MD; Brian Patterson, MD; Michael Lohmeier, MD

\section{A 79-year-old man experienced continued bleeding following canthotomy/cantholysis for a spontaneous retrobulbar hemorrhage.}

$\mathrm{M}$ ost emergency physicians (EPs) encounter several patients a year with hemorrhages due to factor Xa (FXa) inhibitors. Such bleeding may occur in patterns not previously recognized with traditional anticoagulant therapy. Retrobulbar hemorrhage is typically associated with significant facial or orbital trauma, and spontaneous hemorrhage is a very rare cause of orbital compartment syndrome. ${ }^{1}$ Retrobulbar hemorrhage can lead to orbital compartment syndrome due to increased orbital pressure within a closed space. Because orbital compartment syndrome can compromise blood flow to the optic nerve or central retinal artery, it is extremely important to decrease orbital pressure as quickly as possible in affected patients. Therefore, canthotomy/cantholysis should be performed sooner rather than later, as 90 minutes of elevated intraocular pressure (IOP) can lead to permanent vision loss. ${ }^{2}$

Rivaroxaban, one of the relatively new oral anticoagulant agents that inhibit FXa, is used as an alternative therapy to vitamin $\mathrm{K}$ antagonists. The FXa agents have been approved to reduce the risk of stroke in patients with nonvalvular atrial fibrillation (AF). ${ }^{3}$ According to a meta-analysis of rivaroxaban and bleeding risk, rivaroxaban was shown to have no increased risk of major or clinically relevant nonmajor bleeding compared to vitamin $\mathrm{K}$ antagonists. Rivaroxaban was also associated with a significant decrease in fatal bleeding (relative risk, 0.48, 95\%; confidence interval, 0.31 to 0.74$){ }^{4}$

\section{Case Report}

A 79-year-old man with a medical history of hypertension, transient ischemic attacks (TIAs), and AF, for which he was taking rivaroxaban, was referred to our ED by a local rural ED for further evaluation and treatment of a retrobulbar hemorrhage. (The patient's family refused emergency medical services transport from the rural ED.) The patient stated that upon awakening earlier that morning, he felt "pressure" in his right

Dr Brewer is a resident, BerbeeWalsh Department of Emergency Medicine, University of Wisconsin School of Medicine and Public HealthMadison. Dr Mancera is an assistant professor, BerbeeWalsh Department of Emergency Medicine, University of Wisconsin School of Medicine and Public Health-Madison. Dr Patterson is an assistant professor, BerbeeWalsh Department of Emergency Medicine, University of Wisconsin School of Medicine and Public Health-Madison. Dr Lohmeier is an assistant professor, BerbeeWalsh Department of Emergency Medicine, University of Wisconsin School of Medicine and Public Health-Madison.

DOI: 10.12788/emed.2016.0040 
eye and experienced periorbital swelling that continued to worsen throughout the day. He denied any trauma, falls, or strikes to the face or head. The patient's account and history were confirmed by the family members with whom he resided.

During the patient's evaluation at the rural ED, a computed tomography (CT) scan of the head was performed, which demonstrated a retrobulbar hematoma on the right side (See the Figure for an example of a CT scan illustrating a retrobulbar hematoma with proptosis). Since the patient's initial right IOP was $32 \mathrm{~mm} \mathrm{Hg}$ (normal range, $12-22 \mathrm{~mm} \mathrm{Hg}$ ), ophthalmology services at this institution performed a lateral canthotomy. The patient's right IOP postsurgery decreased but remained elevated at $27 \mathrm{~mm} \mathrm{Hg}$. In addition to surgical intervention, he was given oral acetazolamide and timolol. Then, because the patient was hemodynamically stable, he was referred to our institution for further evaluation.

Upon arrival at our ED, the patient reported slow bleeding from the canthotomy

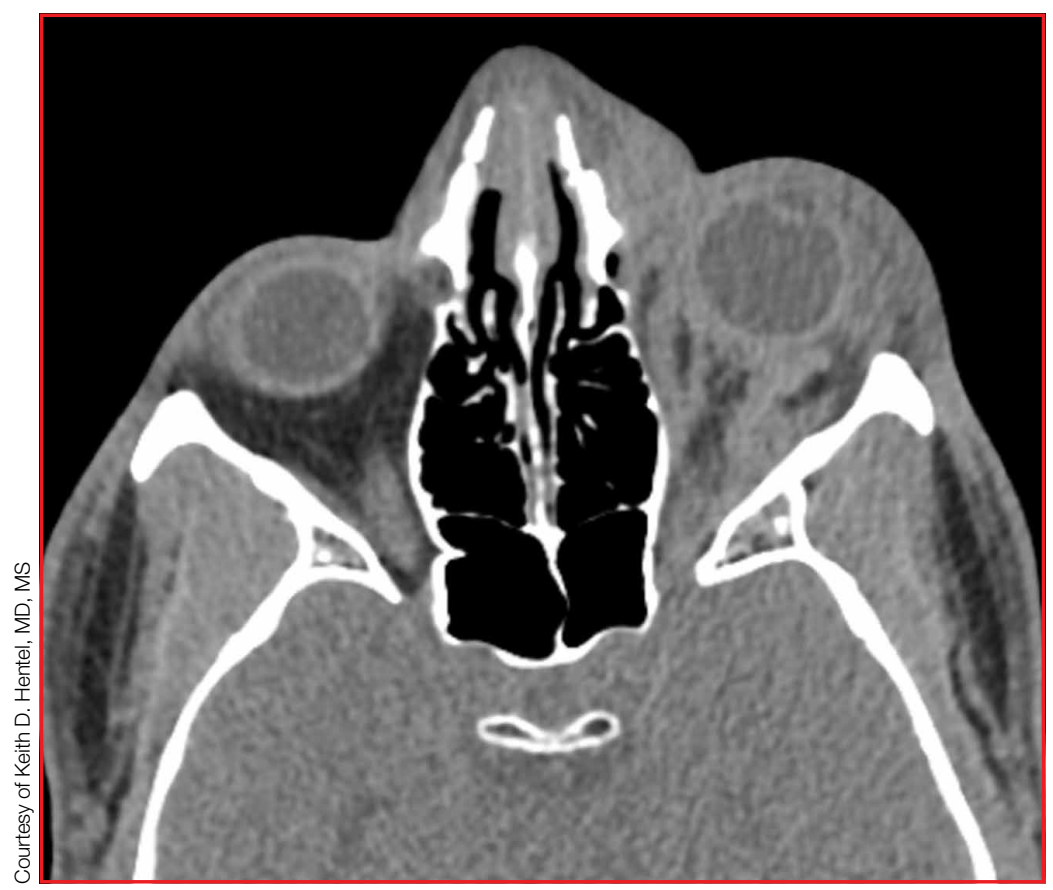

Figure. A head computed tomography scan (not of the patient described in this article) illustrating a left retrobulbar hemorrhage and proptosis. site. He denied any chest pain, shortness of breath, light-headedness, dizziness, or visual changes. Additional history revealed that in addition to taking rivaroxaban, the patient was also on a daily 81-mg aspirin regimen. His vital signs at presentation were: blood pressure (BP), 130/68 mm Hg; heart rate, 75 beats/minute; and respiratory rate, 16 breaths/minute; and temperature, afebrile. Oxygen saturation was $99 \%$ on room air.

Physical examination revealed blood oozing from the right eye at the canthotomy site. There was no other evidence of trauma to the eye or head, and IOP of the right eye was normal at $14 \mathrm{~mm} \mathrm{Hg}$. Laboratory studies revealed a hemoglobin value of $16.8 \mathrm{~g} / \mathrm{dL}$, a hematocrit of $48 \%$, and a white blood cell (WBC) count of $8.8 \times 10^{9} / \mathrm{L}$. The basic metabolic profile, including creatinine, was unremarkable. A type and screen blood pretransfusion compatibility test was also ordered.

Since the patient's ocular hemorrhaging persisted, ophthalmology services were consulted. The ophthalmology examination measured a right IOP of $14 \mathrm{~mm} \mathrm{Hg}$ and a visual acuity of $20 / 200$. The patient's pupils were equal, round, and reactive to light, and a subconjunctival hematoma was noted. The ophthalmologist recommended no further surgical interventions at that time.

Due to the continued ocular bleeding, hematology services were also consulted. The hematologist recommended $50 \mathrm{U} / \mathrm{kg}$ of intravenous (IV) prothrombin complex concentrate (PCC) to reverse the anticoagulatory effects of rivaroxaban. The patient was given one dose of PCC in the ED. Throughout his ED course, the patient did not experience any deterioration of visual acuity. However, during repeated IOP checks, he experienced one episode of vasovagal syncope with a systolic BP in the 70s. The syncope resolved promptly after the patient was placed in a supine position and was given an IV bolus of normal saline fluid. The patient still had oozing at the incision site, and was admitted to the general medicine floor. 


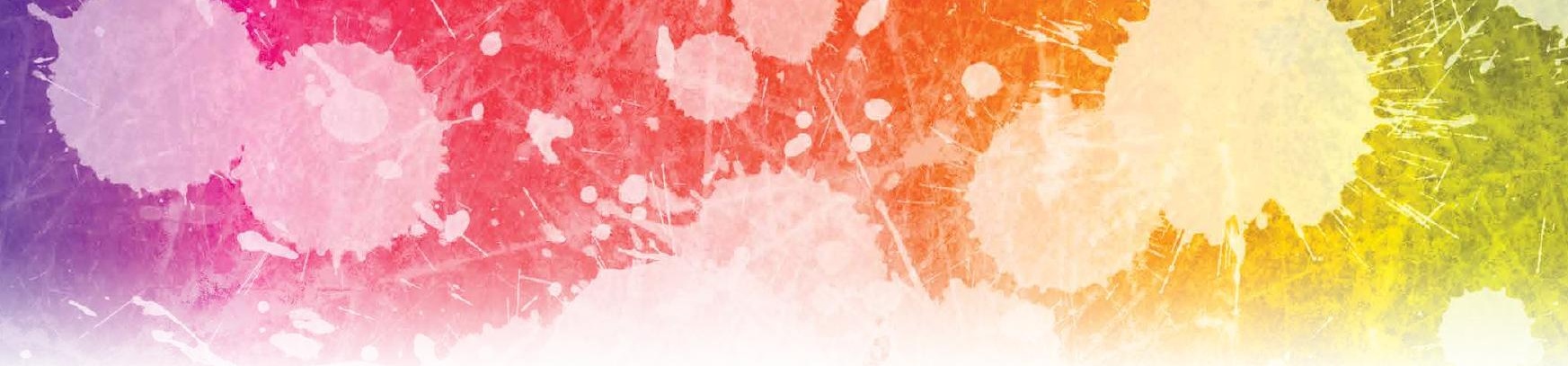

During his inpatient stay, the patient remained hemodynamically stable and did not require transfusion of blood or platelet products. All home anticoagulant medications were discontinued. The patient continued to have some oozing the following morning, and was given an additional dose of IV PCC ( $50 \mathrm{U} / \mathrm{kg}$ ), which resolved the bleeding. He remained hemostatic and, based on his history of AF, he was discharged home on warfarin without bridge therapy. Both rivaroxaban and daily aspirin therapy were discontinued. The lateral canthotomy and cantholysis healed without need for surgical intervention. An ophthalmology follow-up clinic visit 1 week after discharge from the hospital revealed an already self-healed incision without ectropion or retraction and with only mild laxity. Given the patient's history of AF with TIAs while off anticoagulants, the ophthalmologist did not recommend any other surgical intervention that would have required discontinuing the warfarin.

\section{Discussion}

With any retrobulbar hematoma, one must be concerned for orbital compartment syndrome. Orbital hemorrhage is the most common cause of orbital compartment syndrome, usually occurring secondary to trauma, surgery, or retrobulbar injection. In this case, spontaneous hemorrhage due to anticoagulation was believed to be the cause-albeit a rare one-of orbital compartment syndrome. Because the orbital space is enclosed and cannot expand, it is vulnerable to compartment syndrome, and subsequent ischemia can lead to permanent vision impairment or complete loss of vision. ${ }^{5}$ Early recognition and treatment is imperative to preserve vision as an elevated intraorbital pressure for 60 to 100 minutes can lead to permanent visual sequelae.

\section{Management}

Treatment of retrobulbar hemorrhage includes lateral canthotomy and cantholysis, which have been shown to reduce IOP an average of $14.2 \mathrm{~mm} \mathrm{Hg.}{ }^{6}$ In our patient, IOP in the affected eye was reduced by $18 \mathrm{~mm} \mathrm{Hg}$. In addition to the patient's high IOP at presentation, another concern was the continued hemorrhaging from both the incision site and its potential to exacerbate the underlying retrobulbar hematoma. Management of this condition posed a challenge because this patient was taking a newer anticoagulant, for which

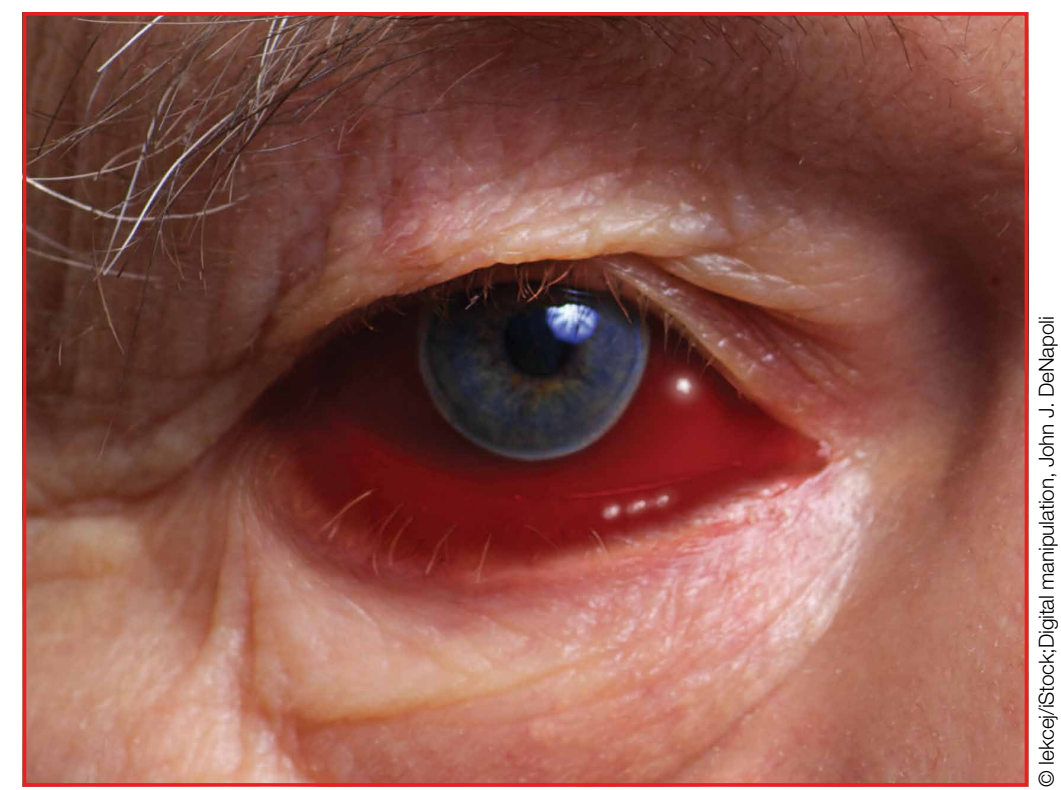

there is currently no specific reversal agent. After consultation with hematology services, the patient was given PCC because small studies have suggested that PCC may reverse rivaroxaban-induced anticoagulation. ${ }^{7}$ While more expensive than fresh frozen plasma, PCC has a high safety profile and should be considered in cases of life-threatening bleeding-especially in patients who have renal failure, as rivaroxaban is renally excreted. The half-life of rivaroxaban is 5 to 9 hours and its effects may last up to 12 hours. An IV dose of $50 \mathrm{U} / \mathrm{kg}$ PCC can be effective in reversing rivaroxaban; this dose can be repeated every 12 hours until hemorrhaging abates or until rivaroxaban is cleared. 


\section{Potential Factor Xa Reversal Agent}

Phase IV trials are underway in the ANNEXA (Andexanet Alfa a Novel Antidote to the Anticoagulant Effects of FXa inhibitors - Apixaban) and ANNEX-R (Andexanet Alfa a Novel Antidote to the Anticoagulant Effects of FXa inhibitors - Rivaroxaban) studies assessing andexanet alpha, an FXa inhibitor reversal agent and potential FXa inhibitor antidote. Andexanet alpha is a decoy protein that binds to FXa inhibitors in the active site, restoring endogenous FXa and reducing anticoagulant activity. ${ }^{8}$ This serves as another promising reversal agent for apixaban, edoxaban, and rivaroxaban. With the development of these new FXa reversal agents, EPs will have more options for reversal of anticoagulation in patients with unique hemorrhagic presentations.

\section{Conclusion}

Rivaroxaban has the potential to replace warfarin as a "novel" oral anticoagulant of choice for multiple indications, especially as more insurance companies cover the use of the FXa inhibitors. As a result of their increased use, the EP is likely to see an increasing number of patients who present with hemorrhagic consequences of the FXa inhibitors, and in turn must be familiar with the properties of this class of anticoagulants- including potential reversal strategies.

Our case of spontaneous retrobulbar hemorrhage may be one of these new patterns of bleeding to be expected from a novel FXa inhibitor. Therefore, it is imperative that EPs consider retrobulbar hemorrhage and other possible bleeding locations in patients on an FXa inhibitor.

\section{References}

1. McAllister AR, Sobel RK, Allen RC. Spontaneous retrobulbar hemorrhage with subsequent orbital compartment syndrome. University of Iowa Health Care Ophthalmology and Visual Sciences Web site. http://www.eyerounds.org/cases/168-orbital-compartment-syndrome.htm. Accessed June 14, 2016.

2. Winterton JV, Patel K, Mizen KD. Review of management options for a retrobulbar hemorrhage. J Oral Maxillofac Surg. 2007;65(2):296-299.

3. Wasserlauf G, Grandi SM, Filion KB, Eisenberg MJ. Meta-analysis of rivaroxaban and bleeding risk. Am J Cardiol. 2013;112(3):454-460.

4. Patel MR, Mahaffey KW, Garg J, et al; ROCKET AF Investigators. Rivaroxaban versus warfarin in nonvalvular atrial fibrillation. $N$ Engl J Med. 2011;365(10):883-891.

5. Kloss BT, Patel R. Orbital compartment syndrome from retrobulbar hemorrhage. Int J Emerg Med. 2010;3(4):521-522.

6. Peak DA. Acute orbital compartment syndrome. Medscape. http://emedicine.medscape.com/ article/799528-overview. Updated November 4, 2015. Accessed June 14, 2016.

7. Eerenberg ES, Kamphuisen PW, Sijpkens MK, Meijers JC, Buller HR, Levi M. Reversal of rivaroxaban and dabigatran by prothrombin complex concentrate: a randomized, placebo-controlled, crossover study in healthy subjects. Circulation. 2011;124(14):1573-1579.

8. Siegal DM, Curnutte JT, Connolly SJ, et al. Andexanet alfa for the reversal of factor Xa inhibitor activity. N Engl J Med. 2015;373(25):2413-2424. 